\title{
Human Fall Down Recognition Using Coordinates Key Points Skeleton
}

https://doi.org/10.3991/ijoe.v18i02.28017

\author{
Mohammed Abduljabbar Ali $\left.{ }^{1}{ }^{凶}\right)$, Abir Jaafar Hussain ${ }^{1,2}$, Ahmed T. Sadiq ${ }^{1,2}$ \\ ${ }^{1}$ Computer Sciences Department, University of Technology, Baghdad, Iraq \\ ${ }^{2}$ School of Computer Sciences and Mathematics, Liverpool John Moores University, \\ Liverpool, England \\ cs.19.21@grad.uotechnology.edu.iq
}

\begin{abstract}
Falls pose a substantial threat to human safety and can quickly result in disastrous repercussions. This threat is particularly true for the elderly, where falls are the leading cause of hospitalization and injury-related death. A fall that is detected and responded to quickly has a lower danger and long-term impact. Many real-time fall detection solutions are available; however, these solutions have specific privacy, maintenance, and proper use issues. Vision-based fall event detection has the benefit of being completely private and straightforward to use and maintain. However, in real-world scenarios, falls are diverse and result in high detection instability. This study proposes a novel vision-based technique for fall detection and analyzes an extracted skeleton to define human postures. OpenPose can be used to get skeletal information about the human body. It identifies a fall using three critical parameters: the center of the value of the head and shoulder coordinates, the critical points of the shoulder coordinates, and the distance between the center of the skeleton's head and the floor with the angle between the center of the shoulders and the ground. Our proposed methodology was effective, with a classification accuracy of $97.7 \%$.
\end{abstract}

Keywords - fall down, human skeleton, skeleton tracking

\section{Introduction}

In the realm of computer vision, understanding and detecting human behavior remains a crucial and challenging task [1]. It is useful in a range of research scenarios, including elder surveillance, intelligent surveillance cameras, human-computer interaction, and analyzing video information [2] [3]. It can be divided into two groups based on the identification objectives: the identification and classification of normal human behavior [4] [5] and the detection and warning of abnormal human behavior [6]. Elderly people are more likely to be affected by falls that result in devastating injuries or death. The most common adverse effects of falls are fractures and longterm illnesses, which result in incapacity, loss of independence, and psychological worry about falling again[7]. Falling puts the elderly at risk for severe or moderate injuries, as well as putting a psychological and financial burden on them and their 
families [8]. In this scenario, it is critical to identify the elderly's falls immediately and effectively to provide emergency aid [9]. In summary, persons who fall and cannot request aid must be identified and treated as soon as possible [7] [10].

Sensor-based and vision-based fall detection systems are both available. The sensor-based system [3] [11] allows inexpensive systems to be built utilizing simple sensors; however, these systems must be connected to a human body. The manufacturing environment requires vision-based systems that do not need to connect to a body [12]. These devices may be portable with a microcontroller attached to the camera for real-time processing and analysis, or they could merely be cameras that send the video stream to a centralized computer for processing [13]. The detection of a moving object continues to be a difficult task [14]. Several traditional methods accomplish detection and classification. Among current approaches, deep learning with neural networks is highly promising in terms of its capability, but quite demanding in terms of the processing time required [15]. This paper provides a new fall detection system. This procedure processes every frame. It uses the 2D Pose Estimation using the Part Affinity Fields skeleton extraction algorithm [16] to obtain the skeletal data file of humans on the screen, which was captured by a surveillance video [17]. Horizontal and vertical coordinates represent each node in a planar coordinate system. The conditions determine the fall behavior and whether a person can stand alone after falling by examining the coordinates of the center of the head to the center of the shoulders the line between the shoulder points, the distance between the center of the head and ground, and the angle of the centerline of the human body with the ground.

Health care employees may utilize our research findings to recognize actions quickly and accurately in real-time from vast amounts of video data from a surveillance system and respond swiftly and effectively in an emergency. In addition, it can improve intellectual awareness of public safety. As a result, reliable human fall detection in public situations has economic and social ramifications.

The remainder of this paper is arranged as follows. The second section contains related work. The third section depicts system models, while Section 4 depicts experimental outcomes. Finally, the fifth section is the conclusion.

\section{Related work}

Since cameras are so common in modern society, they are increasingly utilized to acquire helpful information. They are well-known tools in vision-based fall recognition systems. To identify or avoid falls several studies have used depth thermal sensors, RGB cameras, cameras (Kinect), or even a combination of cameras to monitor body size, head trajectory, or body position changes. Jin Zhang et al. proposed a model of human body position fall recognition named the "five-point inverted pendulum model." This model extracts and constructs the pendulum structure of human posture in complicated natural scenes using an enhanced two-branch multi-stage convolutional neural network model (M-CNN) [7].Weiming Chen et al. used OpenPose to obtain skeleton information from the human body and to detect a fall using three essential parameters: velocity of descent at the core of the hip joint, human body centroid an- 
gles with the floor, and externally rectangular width-to-height ratios. This approach recognizes the fall-down action with a 97 percent success rate [18]. Fan et al. suggested a new vision-based method for detecting falls by analyzing extraction features used to define human body position. The characteristics are put into a directed acyclic graph support vector machine to distinguish four closely related human postures, standing, sitting, crouching, and lying. To detect a fall, it counts the number of occurrences of laying postures in a restricted time interval. After majority voting, immobility verification determines a fall event. According to the results of the trials, recognition of the four postures has an overall accuracy of 97.1 percent, with only 1.0 percent of the postures misclassified as lying positions. A fall detection system achieves up to 95.2 percent fall detection accuracy on a public fall dataset [19]. Liu et al. based their work on Kinect. After extensive testing, the researchers developed a novel fall identification system that quickly and accurately detects human falls. This technique has three parts: movement targets depth of image capture, depth image processing, and target motion behavior identification. The detection method uses the depth map sequence generated by Kinect. Data on fall and bending is obtained and compared in this research. The anti-noise performance of the Otsu algorithm is used to process the depth map. It simplifies the extraction of the physical outlines. After the contour has been removed, a corrosion technique is utilized to secure the edge. The human outer rectangle's aspect ratio, the human body's gravity center, and the inclination degree are then retrieved [20]. Kong et al. To protect older individuals, our study devised an algorithm to recognize dangerous situations in the living room. A depth camera with a Canny filter obtained the contour of the binary picture. The outline image that resulted was then used to identify falling objects. It collects all white pixels in the outline image, calculates their tangent vector angles, and divides them into 15 groups. If the majority of tangent angles are less than 45 degrees, a decline is detected [21]. Rafferty et al. The computer vision algorithms were combined with a thermal vision sensor mounted on the ceiling to detect falls. Traditional approaches have drawbacks, which the innovative solution in this method address. On the other hand, vision-based fall detection also has several drawbacks, including high computation and storage needs to run the real-time algorithm, privacy concerns, and a restricted capture zone that can be monitored. A preliminary investigation of this method yielded encouraging findings with a 68 percent accuracy rate, but it also raised concerns about false positives [22].

\section{$3 \quad$ System modelling}

The five steps in our proposed strategy are: (1) get the human body skeleton information using OpenPose, (2) trace the human body with the skeletal information, (3) evaluate the first decision condition (the $\mathrm{x}$-coordinate of the head center and the center of the shoulders), (4) evaluate the second decision condition (the y-coordinate of the shoulder points), and (5) evaluate the third decision condition (the height ratio of the human head body to the ground and the angle between the center of the shoulders 
with the ground). The procedures for implementing our proposed approach appear in Figure 1.

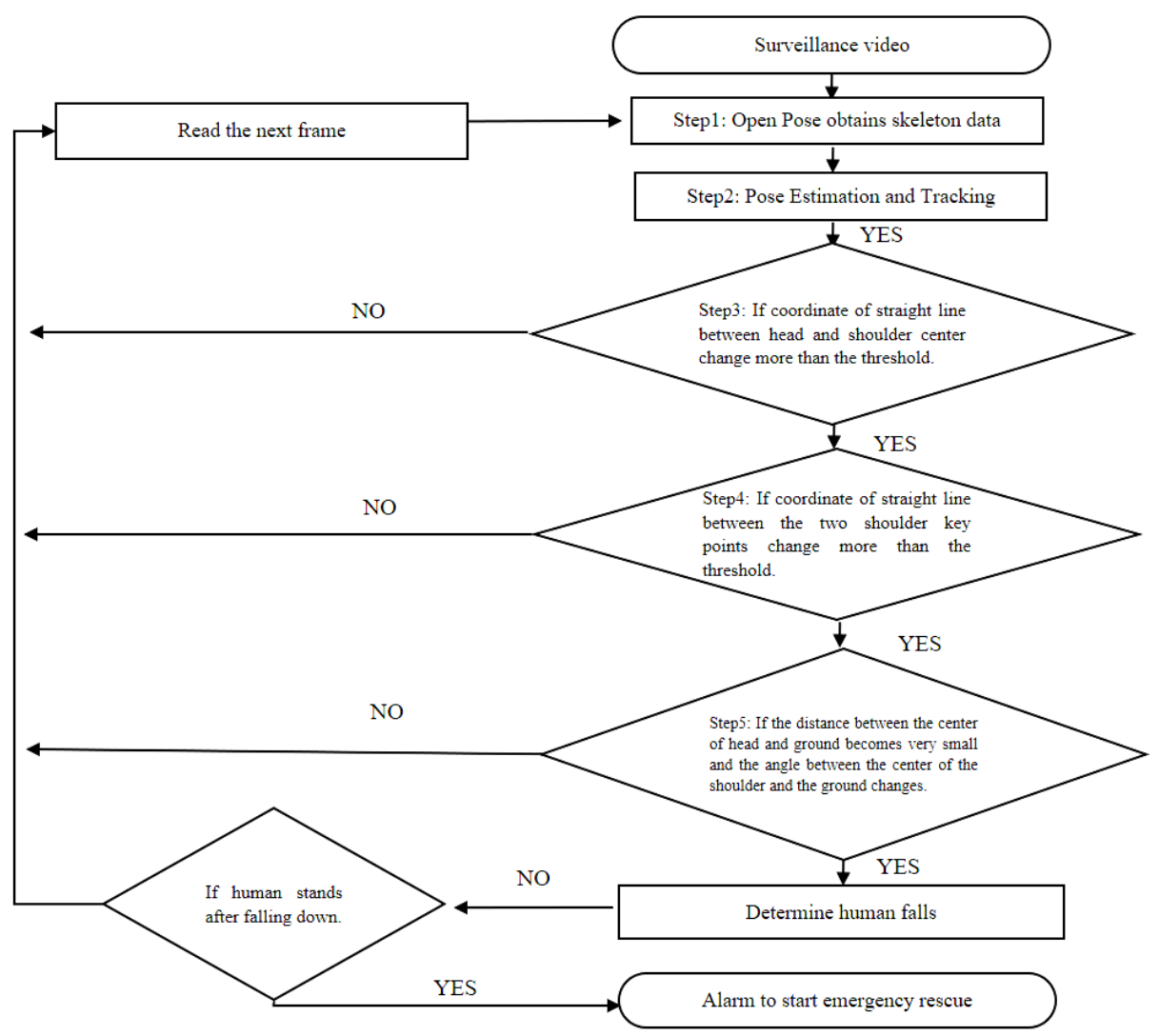

Fig. 1. The workflow of our proposed approach

\subsection{Skeleton data information}

Carnegie Mellon University (CMU) developed the OpenPose human gesture recognition technology, which is based on Convolutional Architecture for Fast Feature Embedding (Caffe) [16]. It depends on supervised learning and convolutional neural networks. In 2017, Carnegie Mellon University researchers released the software for OpenPose's human skeleton detection algorithm, enabling real-time target tracking while the video is being viewed. It can record COCO (Common Objects in Context) human skeletal information and transmit joint information in a color film. The OpenPose human key node recognition system can recognize multi-person skeletal information in real-time. It employs the feature vector affinity parameter to produce a hot spot map of critical human nodes after using the top-down human body attitude estimation approach to locate key spots on the human body. Human movement, finger movement, facial expression, and other posture evaluations are possible 
with OpenPose. It is suitable for both an individual and a large group of people, and it is highly durable. OpenPose obtains the information of human key nodes from the image taken by the surveillance camera. The surveillance film is broken up into frames, each of which depicts a human skeleton. The horizontal and vertical coordinate values indicate each joint point's position information, as well as the accuracy of each joint point. Unfortunately, the precision of several joints' coordinate positions is not perfect. Flaws in the OpenPose algorithm primarily cause this problem, but minor deviations in some crucial areas have little impact on the detection of the entire fall action. Figure 2 depicts the specific joint points corresponding to each joint point number [18].

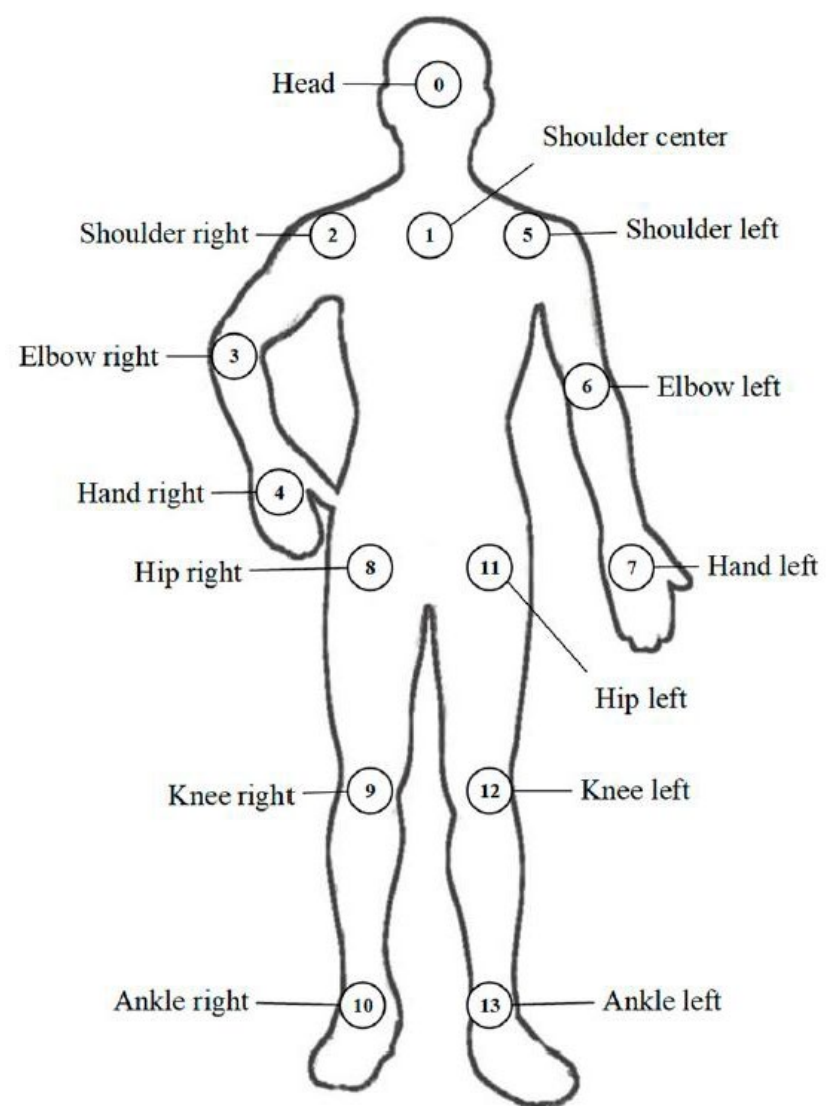

Fig. 2. Node human skeleton [18]

\subsection{Pose tracking}

The posture tracking method follows the pose estimation. After extracting a pedestrian's skeleton model from each image, each individual is connected through the frames to identify their pose for the next step. However, a skeleton model is used in 
an existing method for human pose tracking via actual video. The pedestrian tracking might be handled with a more straightforward approach because they move straight at a modest speed with much less obstruction.

The skeleton model's neck and head locations determine the tracking technique. Compared to other sections of the body, most of the frames can consistently capture the neck and head of the skeleton model. Furthermore, the skeleton model's leg movement is considerably greater than each pedestrian's head and neck movement, making it impossible to utilize as a tracking reference. The technique efficiently differentiates persons from one another and provides them with a unique ID by tracking the upper body of each human. Figure 3 depicts the head and neck movement of a single pedestrian.

Because pedestrians travel short distances between adjacent frames, calculating the distance between each frame's head and neck is a valuable approach to distinguish each individual for tracking. It captures each person's neck and head in a specific structure and analyzes the translation movements of the head and neck as a group across several frames. The data format for each person's neck and head location is:

[person_ID, (x_head, y_head), (x_neck, y_neck), point_ID]

It will be classified as the same person if the $\mathrm{x}$-axis and $\mathrm{y}$-axis of the individual's neck and head are within a particular range.

It saves data for each person to unique files after matching the head and neck coordinates. In one frame, all 14 points of skeleton data, the frame ID, and the person ID are recorded. It will be stored as -1 if the posture estimation output yields no data record because many pedestrians are moving in and out of the video. To properly monitor each person's posture, it must update the array when new people appear in a video and cease capturing skeletal data when a person exits. The method compares the candidate number to the maximum candidate number recorded to supplement the matrix. It expanded the matrix by adding -1 to previous frames and collecting data from the current frame if the candidates exceeded the maximum number. If one individual vanishes from a video, a penalty value must be added to ensure no data is recorded later [23].

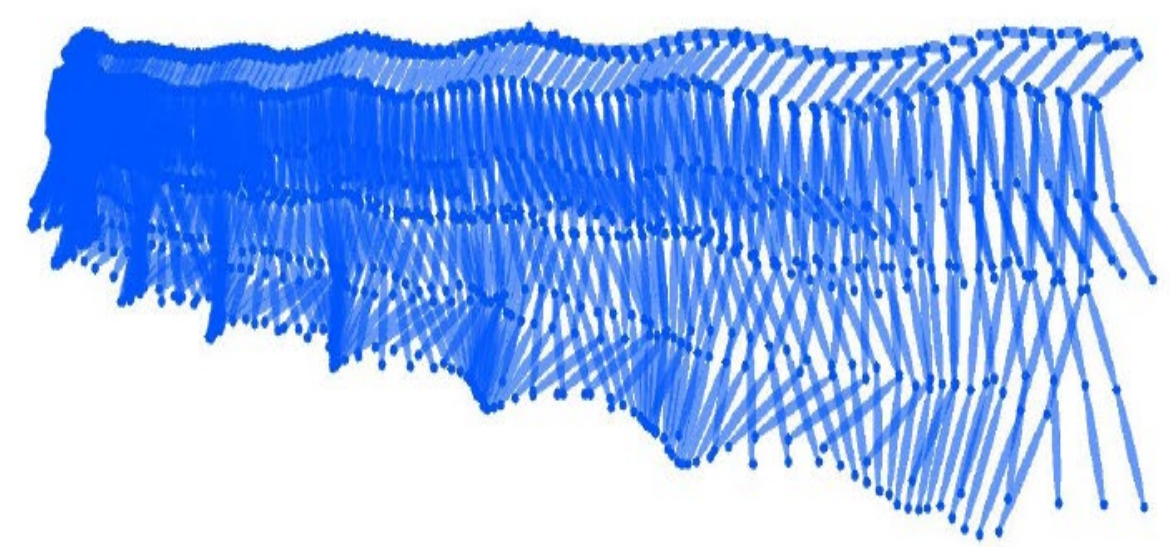

Fig. 3. A single pedestrian's head and neck movement [23] 


\subsection{First step (check and detect the center of head and shoulder direction of key points human body)}

The key points from the center of the human body's head and the shoulder center will change from vertical to horizontal during a quick fall. The coordinates of these two points are obtained for each image frame by processing the combined data provided by OpenPose. In the cases of standing, sitting, and walking, it depicts a straight line connecting them with an equal or convergent $\mathrm{x}$-coordinate. However, the transition from standing to falling is rapid, and it is only detected once every 15 frames. $\mathrm{s} 0$ $=(\mathrm{x} 0, \mathrm{y} 0)$ and $\mathrm{s} 1=(\mathrm{x} 1, \mathrm{y} 1)$ are the center of the human head and neck coordinates, where the X-coordinates of each are essentially similar in value in the standing situation. The $\mathrm{Y}$-coordinate is highly variable. If the $\mathrm{x}$ and $\mathrm{y}$ coordinates exceed a threshold defined in the code, the system signals that the body is starting to fall or tilt. The $y$-axis coordinates of the center of the head and shoulder bodies in the fall are nearly equal.

\subsection{Second step (check coordinates of shoulder key points to $x$-axis directed)}

The process of a sudden fall of a human will change the coordinates of the key points of the skeleton. In the event of a fall, the line connecting the shoulder key points can be determined by scanning in the direction of the y-axis. The value of the $\mathrm{x}$-axis is a straight line between the shoulder points. In the case of standing, there is a big difference in the value of the $\mathrm{x}$ coordinates, but the $\mathrm{y}$-axis is almost equal. While tracing the human skeleton and showing a change in the value of the shoulder $y$-axis in several airless tires, considering that the first condition is met, the result is either the fall or the tilt of the person. So, S2 = (x2, y2) represents the right shoulder key point, and $\mathrm{S} 5=(\mathrm{x} 5, \mathrm{y} 5)$ represents the left shoulder. In the case of a fall, y2 $>\mathrm{y} 5$ or vice versa depending on the direction of the body tilt.

\subsection{Third step (check the distance between the head and floor and that the angle is less than $\mathbf{4 5}$ degrees)}

The distance between the center of a human head and the floor is considerable when standing. During a fall, the body begins to tilt towards the ground. The distance between the center of the head and the ground decreases, and the inclination angle decreases. Using the 2D distance formula in Equation 1, the distance between the center of the head and the ground is determined in Figure 4. The center of the human head in the data obtained from the skeleton is $\mathrm{S} 0$. The ground is determined based on the values of S10 and S13, which represent the position of the points of the ankle of the skeleton when standing. Thus, $\mathrm{s} 0=(\mathrm{x} 0, \mathrm{y} 0), \mathrm{s} 10=(\mathrm{x} 10, \mathrm{y} 10)$, and $\mathrm{s} 13=(\mathrm{x} 13$, y13) respectively. When standing, the distance between the ground and the feet points of the human body is measured. The formula for the distance $\mathrm{d}$, between two points whose coordinates are $(\mathrm{x} 1, \mathrm{y} 1)$ and $(\mathrm{x} 2, \mathrm{y} 2)$ is:

$$
d=|x 2-x 1|+|y 2-y 1|
$$




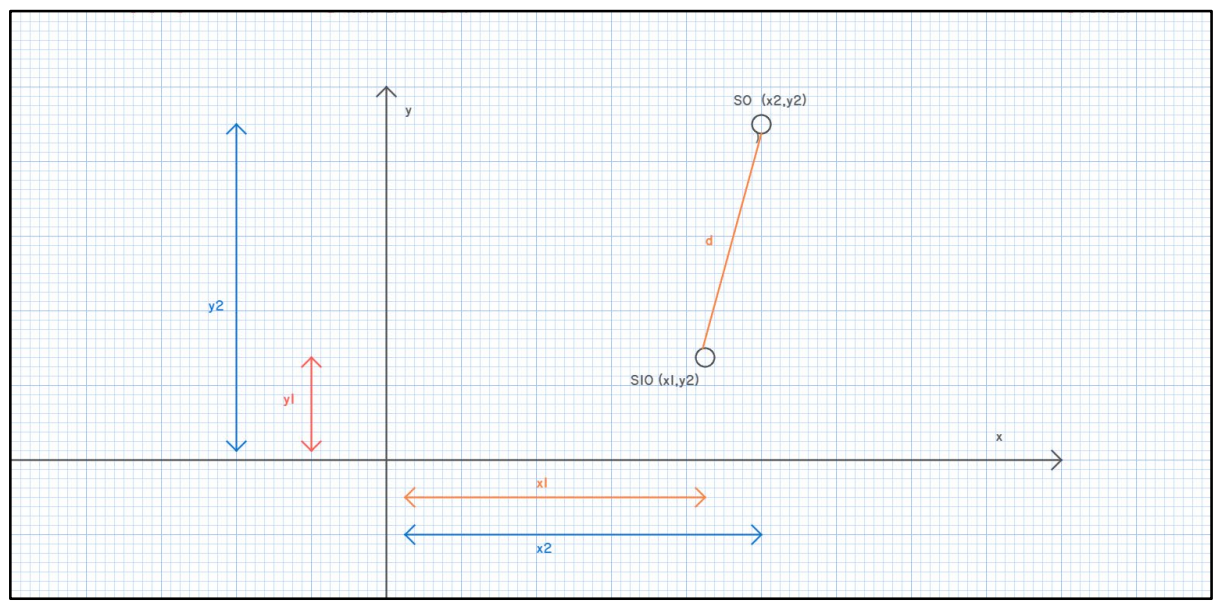

Fig. 4. Finding the distance between two points

Where S0 and S10 represent the center of the head and ankle coordinates, respectively. Next, we will construct a right-angled triangle with S1 and S10 or S13 as the hypotenuse. That is used to find the angle between the S1 center of shoulders and ankle key points S10 or S13, where the angle in standing humans is 45 degrees. When a human begins to lean toward a fall, the angle is less than 45 degrees See Figure 5.

Applying the Pythagorean theorem for the $\triangle \mathrm{ABC}$ :

$$
A B^{2}=A C^{2}+B C^{2}
$$

Here, the vertical distance between the given points is $|\mathrm{y} 2-\mathrm{y} 1|=\mathrm{BC}$.

The horizontal distance between the given points is $|\mathrm{x} 2-\mathrm{x} 1|=\mathrm{AC}$.

And the diagonal distance between the given points $=\mathrm{AB}$.

To find the angle between the center of the human and the ground on a right triangle, follow these steps:

1. The two adjacent lines of a right-angled triangle are known as the adjacent and the hypotenuse.

2. To extract the value of the angle using the cosine.

$$
\cos (\theta)=\frac{\text { Adjacent }}{\text { Hypotenuse }}
$$

3. Using arccosine, find the value of the angle. 


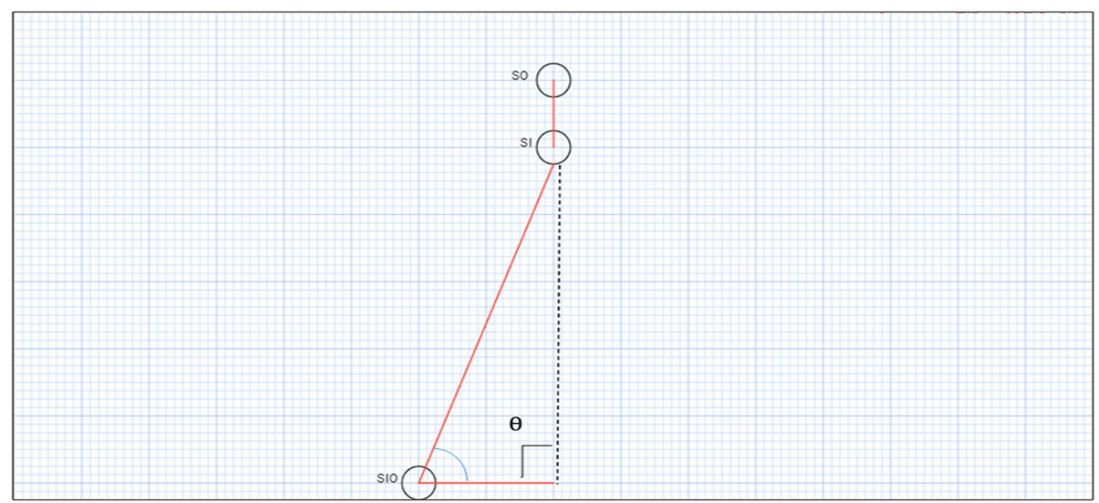

(a)

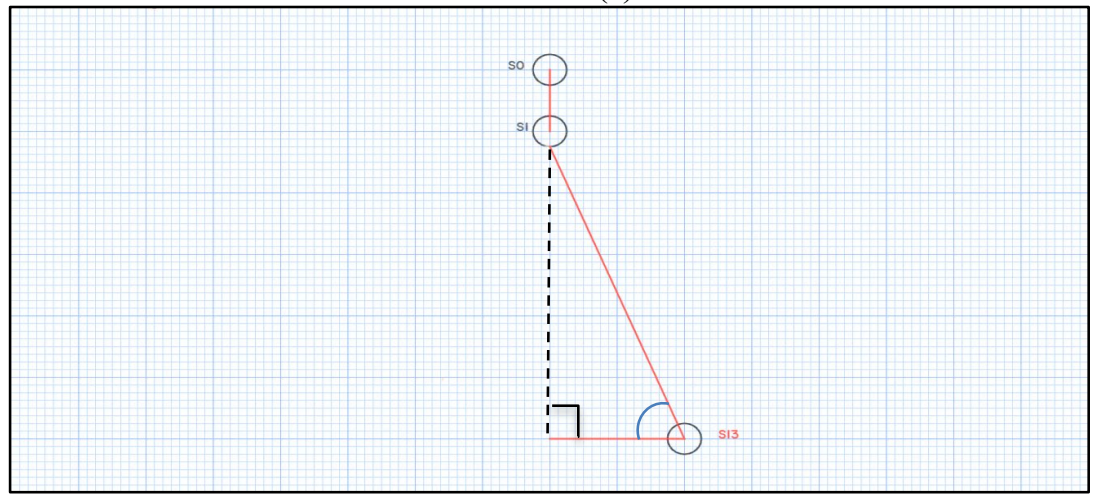

(b)

Fig. 5. Calculate the Angle between the Center of the Shoulders and the Ankles. (a) The angle between the right ankle and the center of the shoulders. (b) The angle between the left ankle and the center of the shoulders

\subsection{Monitoring a human body when standing up after a fall}

Observing the human body after a fall is an important activity. Most fall-down analysis research focuses on the fall detection processes. Standing up after a fall is the opposite of falling. The only difference is that falling is faster than standing up. It is determined that the person has stood up if the coordinates of the center of the head with the center of the chest return to the same value as the $\mathrm{x}$-axis and the angle of inclination of the centerline is approximately equal to 45 degrees. These conditions are based on the tracking algorithm that stored the skeletal information before the fall. The objective of determining whether someone can stand up on their own after falling is to minimize false alarms, as falls may not result in significant injury. 


\section{Experimental results}

\subsection{Data of test description}

The evaluation and the test of our model were based on a sample collected indoors. A single Panasonic HC-MDH2 AVCHD camera, recording in Full-HD (1920 x 1080p), collected the RGB sample. A data set to identify the situation was created that simulates the actual reality. The camera was installed on a tripod in an enterprise building in our trial setup. Figure 6 shows instances of the three types of postures: standing, walking, and falling. Two individuals of varying ages and physical features completed each stance. Each posture was collected at two distinct orientations and distances from the camera to improve the samples and assess our system's capacity to handle size and orientation change. The distances ranged from one to five meters, with an orientation angle of 0 to 360 degrees. Falling activities (falling, standing up after a fall) and daily actions (walking, standing) were gathered in the experiment. A total of 80 activities were recorded, with 60 fall actions and 20 non-falling movements lasting 60-90 seconds each. 400-500 usable video frames were taken as samples out of each stream. The cases of squatting, bending, and sitting, were not taken from the data in the test. Because the third condition of the proposed method requires that the distance between the head and the floor be very small, nearly equal to zero, those cases mentioned were not distinguished.
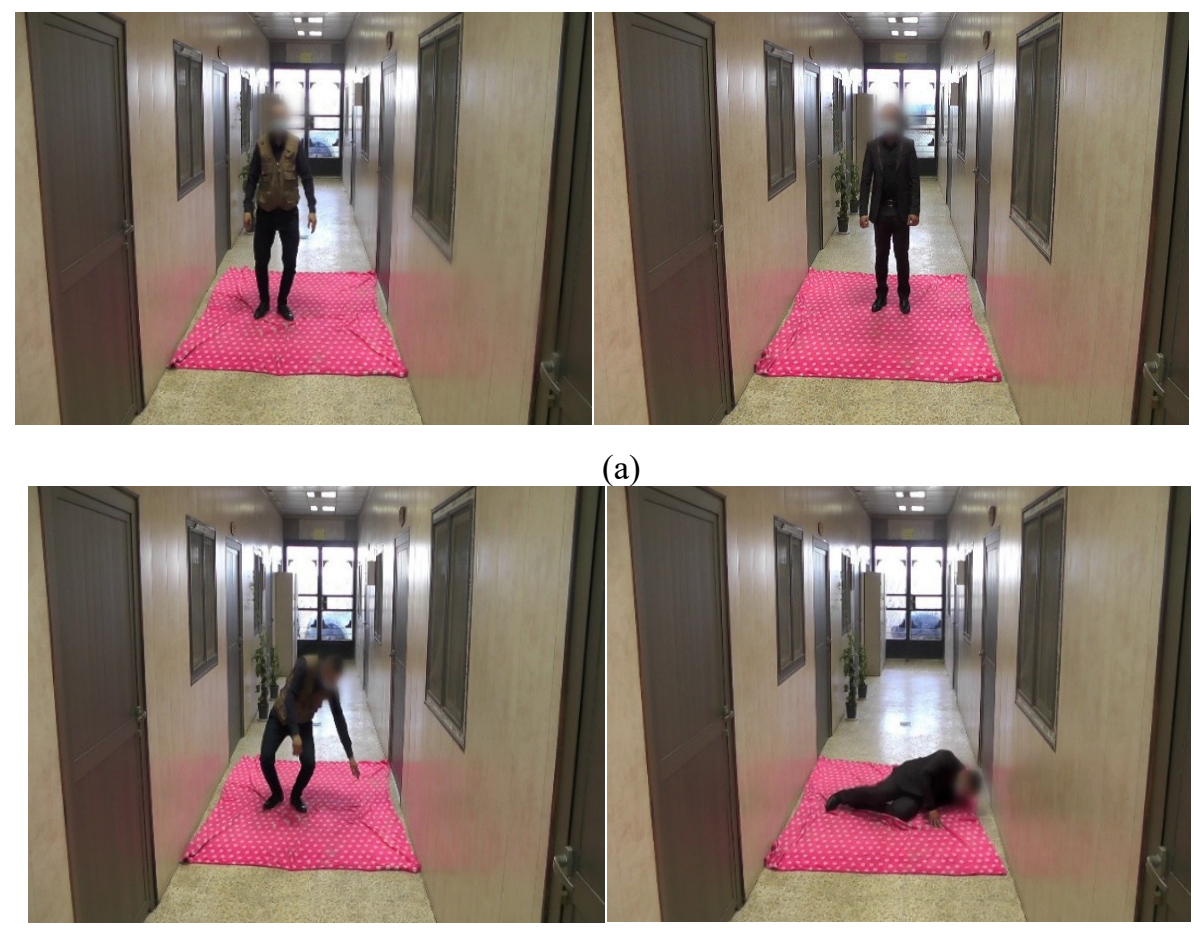

(b) 


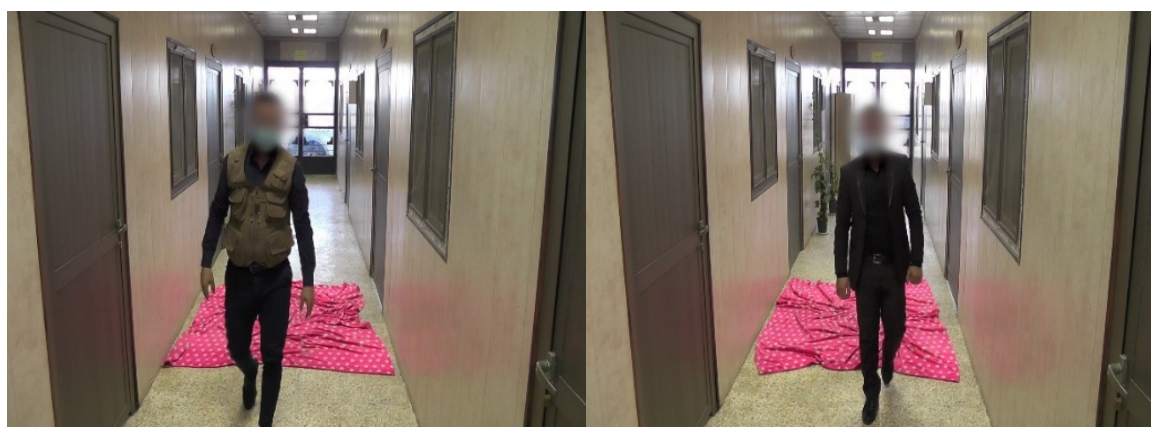

(c)

Fig. 6. Classes of Human Posture (a) The human body in the position of standing. (b) The human body in the position of falling. (c) The human body in the position of walking

\subsection{Evaluation metrics}

The fall test shows the following four possible results. The fall event happened in the first case, and the proposed method correctly detected the fall. In the second case, the fall did not happen, but the method erred in detecting it as a fall. The fall occurs, but the method did not recognize it as a fall in the third case. And in the fourth case, the fall did not occur, and the method did not detect the fall. TP, FP, TN, and FN are the abbreviations for the above four cases.

True positive (TP): a fall occurs and is detected by the device.

A false positive (FP) a fall does not occur, but the equipment detects a fall.

True negative (TN): a fall does not occur, and the equipment does not detect a fall.

A false negative (FN) a fall occurs but is not detected by the equipment.

To evaluate the reaction to these four scenarios, two criteria have been presented.

The ability to detect a fall is referred to as sensitivity:

$$
S E=\frac{T P}{(T P+F N)}
$$

The ability to identify only a fall is known as specificity:

$$
S P=\frac{T N}{(T N+F P)}
$$

Accuracy refers to the ability to distinguish between a fall and no fall:

$$
A C=\frac{(T P+T N)}{(T P+T N+F P+F N)}
$$

According to the above formulas, the following results were obtained for this proposal, sensitivity (98.7\%), specificity (94.6\%), and accuracy (97.7\%). 


\subsection{Comparison}

Table 1 shows a comparison of various fall detection systems. The OpenPose [12] [24] [18] approach is used to recognize the images acquired by the camera, which is quick and straightforward and has a wide range of applications in video-based systems. Vision-based methods are more convenient than other methods. The skeletal information of the human body is obtained using OpenPose, which is both easy and accurate. Our technology was faster than the three methods because it is easy to perform arithmetic operations. The best accuracy was reached, and the experiments proved this because it does not depend on a trained data set. Through this comparison, it is possible to prove that the proposed method was better than the three mentioned methods and the ability to classify the falls in real-time.

Table 1. A comparison of our proposed algorithm with existing techniques for fall detection

\begin{tabular}{|l|c|c|}
\hline & Methods & Accuracy (\%) \\
\hline Sungil Jeong [12] & OpenPose + LSTM & $98.7 \%$ \\
\hline Qingzhen Xu [24] & $\begin{array}{c}\text { OpenPose + Convolutional } \\
\text { neural network }\end{array}$ & $91.7 \%$ \\
\hline Weiming Chen [18] & $\begin{array}{c}\text { OpenPose + } \\
\text { three thresholds }\end{array}$ & $97 \%$ \\
\hline Our proposed & OpenPose + skeleton + tracking + three conditions & $97.8 \%$ \\
\hline
\end{tabular}

\subsection{Analyses of the experimental results}

The viability of the three conditions and final circumstances for standing after a fall are examined in this section. The skeleton key points at the x-axis of the center of the head and the shoulders of the human body in a standing posture are equal, as shown in Figure 7. However, these $\mathrm{x}$ and $\mathrm{y}$ coordinates will change in the first condition, as shown in Figure 8. 
Paper-Human Fall Down Recognition Using Coordinates Key Points Skeleton

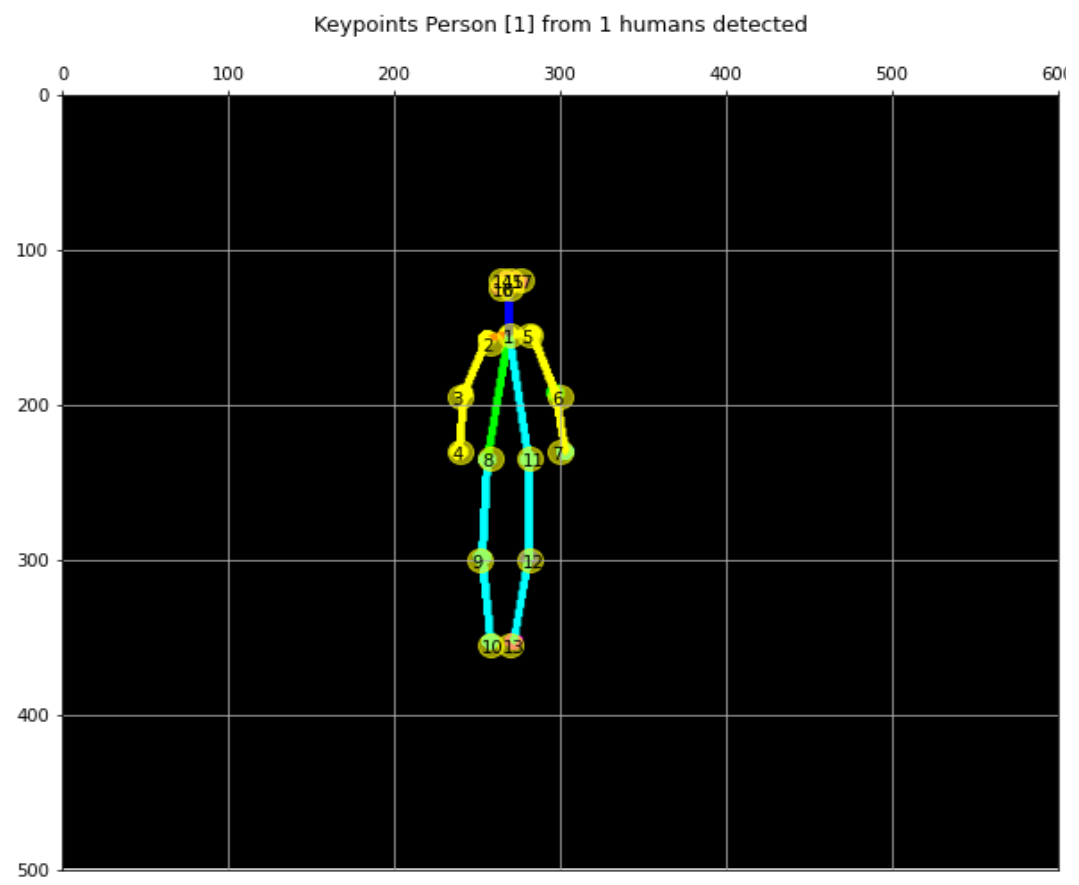

Fig. 7. The key point coordinates of the center of head and shoulders are equal Keypoints Person [1] from 1 humans detected

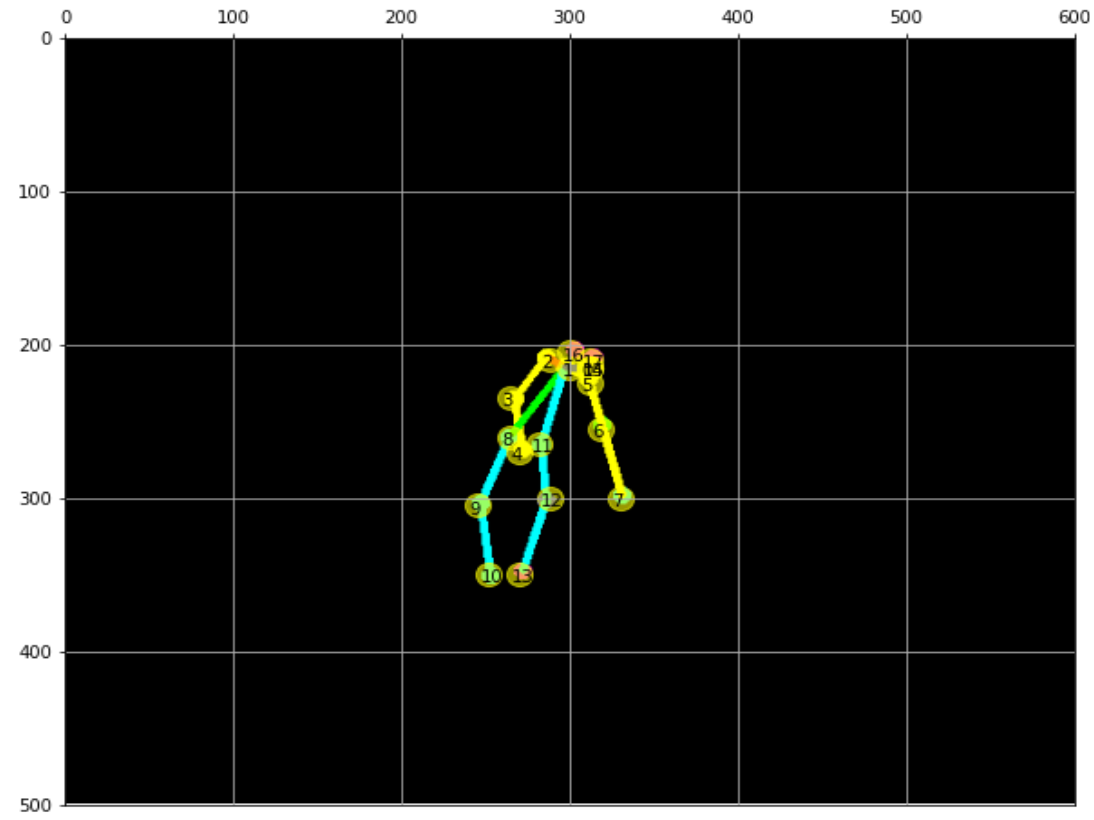

Fig. 8. The key points coordinates of the center of the head and shoulders change 
As shown in Figure 9, when a scan is done in the x-direction, it shows the shoulder points, and the rest of the connecting points appear in the direction of the y-coordinate scan. This arrangement is the case of standing. However, when the body begins to tilt towards a fall, the appearance of these connecting points is reversed, as in Figure 10.
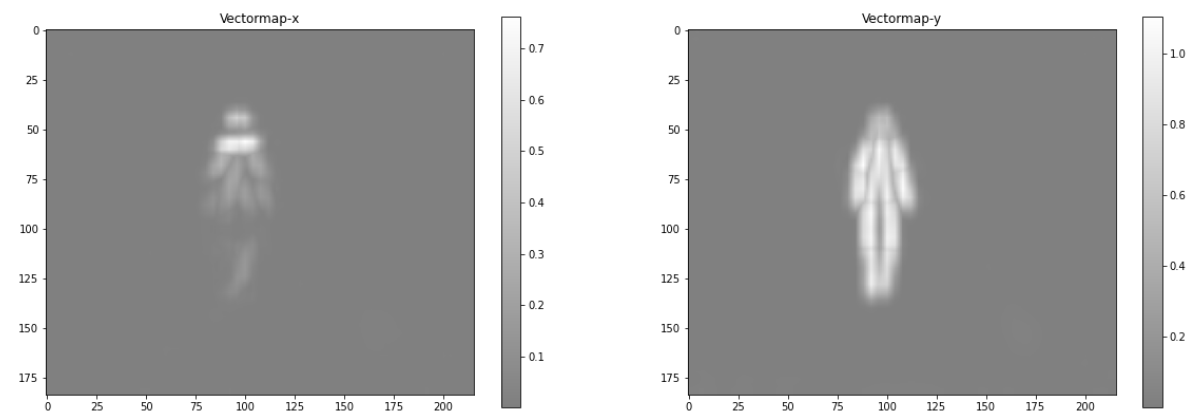

Fig. 9. Vector of the scan of the $\mathrm{X}$-axis and $\mathrm{Y}$-axis
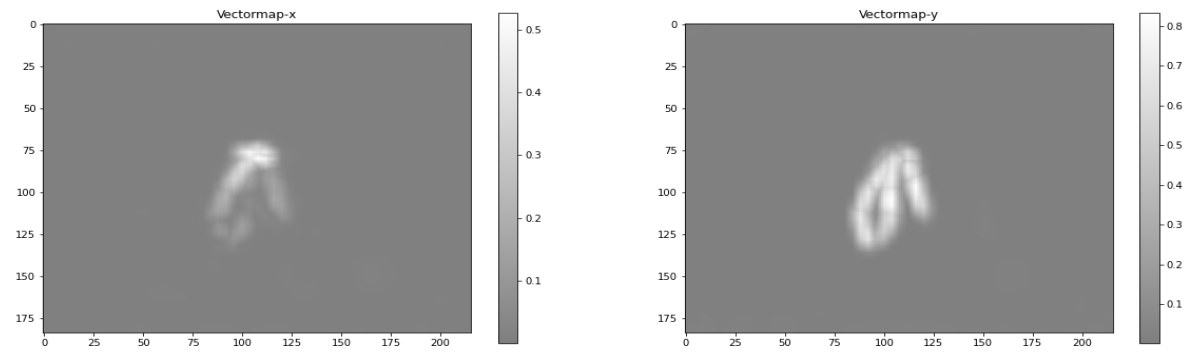

Fig. 10.The Change in the direction of the vector when falling

\section{Conclusions}

In an aging society, a fall is one of the most severe public health risks. Fall detection requires the determination of the features of the fall movement. We present a new solution to this problem in this study. First, the data of human joint points are obtained using the OpenPose method to analyze video captured by a surveillance camera. Three criteria are then used to detect the falling motion: checking and detecting the center of the head and shoulder direction key points, checking coordinates of shoulder key points in the $\mathrm{x}$-axis direction, and a minimum distance between the human head and the floor with an angle less than 45 degrees. Finally, it detects if the subject stands up after the fall. Based on the detection of falls and individuals' circumstances, standing up is viewed as a reverse process to falling. Experiments proved that this strategy works and that it produces the best results. The accuracy is $97.7 \%$, the sensitivity is $98.7 \%$, and the specificity is $94.6 \%$. 


\section{$6 \quad$ References}

[1] W. M. Salih Abedi, I. Nadher, and A. T. Sadiq, "Modification of deep learning technique for face expressions and body postures recognitions," Int. J. Adv. Sci. Technol., vol. 29, no. 3 Special Issue, pp. 313-320, 2020.

[2] A. T. Saadeq, E. K. Jabbar, and N. J. Ibrahim, "Human detection and Recognition System," vol. 5, no. 1, pp. 28-51, 2015.

[3] W. M. S. Abedi, I. Nadher, and A. T. Sadiq, "Modified deep learning method for body postures recognition," Int. J. Adv. Sci. Technol., vol. 29, no. 2, pp. 3830-3841, 2020.

[4] H. Rahmani, A. Mian, and M. Shah, "Learning a Deep Model for Human Action Recognition from Novel Viewpoints," IEEE Trans. Pattern Anal. Mach. Intell., vol. 40, no. 3, pp. 667-681, Mar. 2018. https://doi.org/10.1109/TPAMI.2017.2691768

[5] H. H. Phan, N. S. Vu, V. L. Nguyen, and M. Quoy, "Action recognition based on motion of oriented magnitude patterns and feature selection," IET Comput. Vis., vol. 12, no. 5, pp. 735-743, 2018. https://doi.org/10.1049/iet-cvi.2017.0282

[6] D. Jin, S. Zhu, S. Wu, and X. Jing, "Sparse Representation and Weighted Clustering Based Abnormal Behavior Detection," Proc. - Int. Conf. Pattern Recognit., vol. 2018-Augus, pp. 1574-1579, 2018. https://doi.org/10.1109/ICPR.2018.8545794

[7] J. Zhang, C. Wu, and Y. Wang, "Human fall detection based on body posture spatiotemporal evolution," Sensors (Switzerland), vol. 20, no. 3. 2020. https://doi.org/10.3390/s2 0030946

[8] N. A. Jasim and H. T. S. ALRikabi, "Design and Implementation of Smart City Applications Based on the Internet of Things," Int. J. Interact. Mob. Technol., vol. 15, no. 13, pp. 4-15, 2021. https://doi.org/10.3991/ijim.v15i13.22331

[9] F. S. Hameed, Hasan M. Alwan, and Qasim A. Ateia, "Pose Estimation of Objects Using Digital Image Processing for Pick-and-Place Applications of Robotic Arms," Eng. Technol. J., vol. 38, no. 5A, pp. 707-718, May 2020, doi: 10.30684/etj.v38i5a.518.

[10] M. L. Al-dabag, H. T. S. ALRikabi, and R. R. O. Al-Nima, "Anticipating Atrial Fibrillation Signal Using Efficient Algorithm," Int. J. online Biomed. Eng., vol. 17, no. 2, pp. 106-120, 2021. https://doi.org/10.3991/ijoe.v17i02.19183

[11] R. S. Hsiao, T. X. Chen, M. A. Bitew, C. H. Kao, and T. Y. Li, "Sleeping posture recognition using fuzzy c-means algorithm," Biomed. Eng. Online, vol. 17, no. S2, p. 157, Nov. 2018. https://doi.org/10.1186/s12938-018-0584-3

[12] S. Jeong, S. Kang, and I. Chun, "Human-skeleton based Fall-Detection Method using LSTM for Manufacturing Industries," 34th Int. Tech. Conf. Circuits/Systems, Comput. Commun. ITC-CSCC 2019, no. June 2019, 2019. https://doi.org/10.1109/ITC-CSCC.2019. $\underline{8793342}$

[13] S. Memon, S. Bhatti, L. A. Thebo, M. M. B. Talpur, and M. A. Memon, "A Video based Vehicle Detection, Counting and Classification System," Int. J. Image, Graph. Signal Process., vol. 10, no. 9, pp. 34-41, 2018. https://doi.org/10.5815/ijigsp.2018.09.05

[14] A. Raychaudhuri, S. Maity, A. Chakrabarti, and D. Bhattacharjee, Detection of Moving Objects in Video Using Block-Based Approach. Springer Singapore, 2019. https://doi.org/ 10.1007/978-981-10-8974-9 8

[15] S. S. Mahmood and L. J. Saud, "An Efficient Approach for Detecting and Classifying Moving Vehicles in a Video Based Monitoring System," Eng. Technol. J., vol. 38, no. 6A, pp. 832-845, 2020. https://doi.org/10.30684/etj.v38i6a.438

[16] Z. Cao, T. Simon, S.-E. Wei, and Y. Sheikh, "Realtime Multi-person 2D Pose Estimation Using Part Affinity Fields," in 2017 IEEE Conference on Computer Vision and Pattern 
Recognition (CVPR), Jul. 2017, no. April, pp. 1302-1310. https://doi.org/10.1109/CVPR.2 $\underline{017.143}$

[17] A. A. Abdulhussein and F. A. Raheem, "Hand Gesture Recognition of Static Letters American Sign Language (ASL) Using Deep Learning," Eng. Technol. J., vol. 38, no. 6A, pp. 926-937, Jun. 2020. http://dx.doi.org/10.30684/etj.v38i6A.533

[18] W. Chen, Z. Jiang, H. Guo, and X. Ni, "Fall Detection Based on Key Points of HumanSkeleton Using OpenPose,” Symmetry (Basel)., vol. 12, no. 5, p. 744, May 2020. https:// doi.org/10.3390/sym12050744

[19] K. Fan, P. Wang, Y. Hu, and B. Dou, "Fall detection via human posture representation and support vector machine," Int. J. Distrib. Sens. Networks, vol. 13, no. 5, 2017. https://doi.or $\mathrm{g} / 10.1177 / 1550147717707418$

[20] Y. Liu, N. Wang, C. Lv, and J. Cui, "Human body fall detection based on the Kinect sensor," Proc. - 2015 8th Int. Congr. Image Signal Process. CISP 2015, no. Cisp, pp. 367371, 2016. https://doi.org/10.1109/CISP.2015.7407906

[21] X. Kong, L. Meng, and H. Tomiyama, "Fall detection for elderly persons using a depth camera," Int. Conf. Adv. Mechatron. Syst. ICAMechS, vol. 2017-Decem, pp. 269-273, 2018. https://doi.org/10.1109/ICAMechS.2017.8316483

[22] J. Rafferty, J. Synnott, C. Nugent, G. Morrison, and E. Tamburini, "Fall detection through thermal vision sensing," Lect. Notes Comput. Sci. (including Subser. Lect. Notes Artif. Intell. Lect. Notes Bioinformatics), vol. 10070 LNCS, pp. 84-90, 2016. https://doi.org/10. 1007/978-3-319-48799-1_10

[23] Q. Deng, R. Tian, and Y. Chen, "Skeleton model-based pose tracking and behavior recognition for pedestrian \& cyclists from vehicle scene camera in urban area," pp. 12931298, 2018.

[24] Q. Xu, G. Huang, M. Yu, and Y. Guo, "Fall prediction based on key points of human bones," Phys. A Stat. Mech. its Appl., vol. 540, no. xxxx, p. 123205, 2020. https://doi.org/ 10.1016/j.physa.2019.123205

\section{$7 \quad$ Authors}

Mohammed Abduljabbar Ali received the BSc and MSc degrees in Computer Sciences in 2003 and 2017, respectively from the University of technology. his research interests are security of data, Encryption algorithms, computer vision and machine learning. (Department Computer Sciences, University of Technology, Baghdad, Iraq).

Ahmed T. Sadiq is a Professor in the Computer Science Department-University of Technology-Iraq. He received a B.Sc., M.Sc. \& Ph. D. degree in Computer Science from the University of Technology, Computer Science Department, Iraq, 1993, 1996 \& 2000 respectively. He is Professor in A.I. since 2014. His research interests in Artificial intelligence, data security, patterns recognition \& data mining.

Abir Hussain is a professor of Biomedical Science and a member of the Applied Computing Research Group at the Faculty of Engineering and Technology. She completed her PhD study at The University of Manchester (UMIST), UK in 2000 with a thesis title Polynomial Neural Networks for Image and Signal Processing. She has published numerous referred research papers in conferences and Journal in the research areas of Neural Networks, Signal Prediction, Telecommunication Fraud Detection and Image Compression. She has worked with higher order and recurrent neural 
networks and their applications to e-health and medical image compression techniques. She has developed with her research students a number of recurrent neural network architectures. Her research has been published in a number of high esteemed and high impact journals such as the Expert Systems with Applications, PloS ONE, Electronic Letters, Neuro computing, and Neural Networks and Applications.

Article submitted 2021-11-01. Resubmitted 2021-12-16. Final acceptance 2021-12-18. Final version published as submitted by the authors. 